\title{
Linear Forests and Ordered Cycles
}

\author{
Guantao Chen \\ Georgia State University \\ Atlanta, GA \\ Michael S. Jacobson \\ University of Louisville \\ Louisville, KY 40292
}

\author{
Ralph J. Faudree \\ University of Memphis \\ Memphis, TN 38152 \\ Linda Lesniak \\ Drew University \\ Madison, NJ 07940
}

\author{
Ronald J. Gould \\ Emory University \\ Atlanta, GA 30322 \\ Florian Pfender \\ Emory University \\ Atlanta, GA 30322
}

\begin{abstract}
A collection $L=P^{1} \cup P^{2} \cup \cdots \cup P^{t}(1 \leq t \leq k)$ of $t$ disjoint paths, $s$ of them being singletons with $|V(L)|=k$ is called a $(k, t, s)$-linear forest. A graph $G$ is $(k, t, s)$ ordered if for every $(k, t, s)$-linear forest $L$ in $G$ there exists a cycle $C$ in $G$ that contains the paths of $L$ in the designated order as subpaths. If the cycle is also a hamiltonian cycle, then $G$ is said to be $(k, t, s)$-ordered hamiltonian. We give sharp sum of degree conditions for nonadjacent vertices that imply a graph is $(k, t, s)$-ordered hamiltonian.
\end{abstract}

\section{Introduction}

Over the years hamiltonian graphs have been widely studied. A variety of related properties have also been considered. Some of the properties are weaker, for example traceability in graphs, while others are stronger, for example hamiltonian connectedness. Recently a new strong hamiltonian property was introduced in [7] and further studied in [5], [2], and [3].

We say a graph $G$ on $n$ vertices, $n \geq 3$ is $k$-ordered for an integer $k, 1 \leq k \leq n$, if for every sequence $S=\left(x_{1}, x_{2}, \ldots, x_{k}\right)$ of $k$ distinct vertices in $G$, there exists a cycle that contains all the vertices of $S$ in the designated order. A graph is $k$-ordered hamiltonian if for every sequence $S$ of $k$ vertices there exists a hamiltonian cycle which encounters $S$ in its designated order.

$\mathrm{Hu}$, Tian and Wei [4] considered a different question; when is it possible to find a long cycle passing through a collection of paths?

In this paper we combine these two ideas. In order to treat this in generality, we say $L$ is a $(k, t, s)$-linear forest if $L$ is a collection $L=P^{1} \cup P^{2} \cup \cdots \cup P^{t}(1 \leq t \leq k)$ of $t$ disjoint paths, $s$ of them being singletons such that $|V(L)|=k$. A graph $G$ is $(k, t, s)$-ordered if for every $(k, t, s)$-linear forest $L$ in $G$ there exists a cycle $C$ in $G$ that contains the paths of $L$ in the designated order as subpaths. Further, if the paths of $L$ are each oriented and $C$ can be chosen to encounter the paths of $L$ in the designated order and according to the designated orientation on each path, then we say $G$ is strongly $(k, t, s)$-ordered. If $C$ is a hamiltonian cycle then we say $G$ is $(k, t, s)$-ordered hamiltonian and strongly $(k, t, s)$-ordered hamiltonian, respectively. Note that saying $G$ is $(s, s, s)$-ordered is the same as saying $G$ is $s$-ordered. 
We will think of all cycles being directed. For a cycle $C$ and vertices $x, y \in V(C)$, we denote the $x-y$ path on $C$ following the direction of $C$ by $x C y$.

As usual, we will denote the minimum degree of a graph $G$ by $\delta(G)$, and the minimum degree sum of two non adjacent vertices in a graph $G$ by $\sigma_{2}(G)$.

We will say that a graph $G$ on at least $2 k$ vertices is $k$-linked, if for every vertex set $T=\left\{x_{1}, x_{2}, \ldots, x_{k}, y_{1}, y_{2}, \ldots, y_{k}\right\}$ of $2 k$ vertices, there are $k$ disjoint $x_{i}-y_{i}$ paths. The property remains the same if we allow repetition in $T$, and ask for $k$ internally disjoint $x_{i}-y_{i}$ paths. Thus, as an easy consequence, every $k$-linked graph is $k$-ordered and $(2 k-s, k, s)$ ordered.

An important theorem about $k$-linked graphs is the following theorem of Bollobás and Thomason [1]:

Theorem 1 Every 22k-connected graph is $k$-linked.

The following lemmas will be used later.

Lemma 1 If a $2 k$-connected graph $G$ has a k-linked subgraph $H$, then $G$ is $k$-linked.

Proof: Let $T=\left\{x_{1}, x_{2}, \ldots, x_{k}, y_{1}, y_{2}, \ldots, y_{k}\right\}$ be a set of $2 k$ vertices in $V(G)$. Since $G$ is $2 k$-connected, there are $2 k$ disjoint paths from $T$ to $V(H)$. Choose the paths from $T$ to $V(H)$ such that each path contains exactly one element of $V(H)$ (if $x_{i} \in T \cap V(H)$ then the corresponding path consists only of this one vertex). Now we can connect these paths in the desired way inside $H$, since $H$ is $k$-linked.

Lemma 2 If $G$ is a graph, $v \in V(G)$ with $d(v) \geq 2 k-1$, and if $G-v$ is k-linked, then $G$ is $k$-linked.

Proof: Let $T=\left\{x_{1}, x_{2}, \ldots, x_{k}, y_{1}, y_{2}, \ldots, y_{k}\right\}$ be a set of $2 k$ vertices in $V(G)$. If $v \notin T$, we can find disjoint $x_{i}-y_{i}$ paths inside $G-v$. Thus we may assume that $v=x_{1}$. If $y_{1} \in N(v)$, we can find disjoint $x_{i}-y_{i}$ paths for all $i \geq 2$ in $G-v-y_{1}$, since $G-v-y_{1}$ is $(k-1)$-linked. Adding the path $v y_{1}$ completes the desired set of paths in $G$. If $y_{1} \notin N(v)$, then there exists a vertex $x_{1}^{\prime} \in N(v)-T$, since $d(v) \geq 2 k-1$. We can find disjoint $x_{i}-y_{i}$ paths for $i \geq 2$ and a $x_{1}^{\prime}-y_{1}$ path in $G-v$, which we can then extend to an $x_{1}-y_{1}$ path in $G$.

Further, we will use a Theorem of Mader [6] about dense graphs:

Theorem 2 Every graph $G$ with $|V(G)|=n \geq 2 k-1$, and $|E(G)| \geq(2 k-3)(n-k+1)+1$ has a k-connected subgraph.

Corollary 3 Every graph $G$ with $|V(G)|=n \geq 2 k-1$, and $|E(G)| \geq 2 k n$ has a $k$-connected subgraph.

\section{Degree Conditions}

In this section we examine minimum degree conditions sufficient to insure a graph is either $(k, t, s)$-ordered hamiltonian or strongly $(k, t, s)$-ordered hamiltonian. Sharp results for $s=$ $t=k$ were shown in [5], [2] and [3]: 
Theorem 4 [5] Let $k \geq 2$ be a positive integer and let $G$ be a graph of order $n$, where $n \geq 11 k-3$. Then $G$ is $k$-ordered hamiltonian if $\delta(G) \geq\left\lceil\frac{k}{2}\right\rceil+\left\lfloor\frac{n}{2}\right\rfloor-1$.

Theorem 5 [3] Let $k \geq 3$ be a positive integer and let $G$ be a graph of order $n \geq 2 k$. If $\sigma_{2}(G) \geq n+\frac{3 k-9}{2}$, then $G$ is k-ordered hamiltonian.

As a first step, we prove the following theorem:

Theorem 6 Let $s, t, k$ be integers with $0 \leq s<t<k$ or $s=t=k \geq 3$. If $G$ is a (strongly) $(k, t, s)$-ordered graph on $n \geq k$ vertices with

$$
\sigma_{2}(G) \geq\left\{\begin{array}{ll}
n+k-t & \text { if } s=0 \\
n+k-t+s-1 & \text { if } s>0
\end{array},\right.
$$

then $G$ is (strongly) $(k, t, s)$-ordered hamiltonian.

As a corollary, we obtain the following theorem.

Theorem 7 For $k \geq 1$ and $1 \leq t \leq k$, if $G$ is a (strongly) $(k, t, s)$-ordered graph on $n \geq k$ vertices with $\delta(G) \geq \frac{n+k-t+s}{2}$, then $G$ is (strongly) $(k, t, s)$-ordered hamiltonian.

In the same spirit, we will prove another theorem, which is not needed for our main result, Theorem 10.

Theorem 8 Let $s, t, k$ be integers with $1<t / 2<s \leq t \leq k$. If $G$ is a (strongly) $(k, t, s)$ ordered graph on $n \geq 11 k$ vertices with

$$
\sigma_{2}(G) \geq n+k-\frac{t+3}{2}
$$

then $G$ is (strongly) $(k, t, s)$-ordered hamiltonian.

Proof of Theorem 6 and Theorem 8: Since $G$ is (strongly) $(k, t, s)$-ordered, we may choose a longest cycle $C$ containing the paths of a given $(k, t, s)$-linear forest $L$ in the designated order and with the designated orientations (if there are any) on each path. We need to show that $C$ is hamiltonian.

Let $L=P^{1} \cup P^{2} \cup \ldots \cup P^{t}$, and $x_{1}, \ldots, x_{t}, y_{1}, \ldots, y_{t} \in V(C)$, such that $P^{i}=x_{i} C y_{i}$ for all $1 \leq i \leq t$. Note that $x_{i}=y_{i}$ if $P_{i}$ is a singleton. Let $R^{i}=y_{i} C x_{i+1}$ for $1 \leq i \leq t-1$, and $R^{t}=y_{t} C x_{1}$. Let $R=\bigcup_{i} R^{i}$.

Suppose $C$ is not hamiltonian and let $H$ be a component of $G-C$.

Claim 1 No $R^{i}$ contains more than one vertex adjacent to $H$.

Suppose there exists an interval $R^{i}$ with at least two vertices adjacent to $H$. Without loss of generality we may assume that $R^{1}$ is such an interval. Pick two of these vertices $v_{1}, v_{2}$ such that there are no other adjacencies of $H$ in $v_{1} C v_{2} \subset R^{1}$. Note that $r=\left|v_{1} C v_{2}\right|-2 \geq 1$, otherwise $C$ can be extended by at least one vertex.

Let $u_{1} \in N\left(v_{1}\right) \cap H$, let $u_{2} \in N\left(v_{2}\right) \cap H$. Note that we allow $u_{1}=u_{2}$. Consider now $X=\left(N\left(u_{1}\right) \cup N\left(u_{2}\right)\right) \cap C$. There cannot be two vertices consecutive on $R$ in $X$, otherwise $C$ can be extended by at least one vertex. Further, $X$ does not contain any vertices of 
$v_{1}^{+} C v_{2}^{-}$by our choice of $v_{1}, v_{2}$. Note that $R \backslash v_{1}^{+} C v_{2}^{-}$consists of $t-s+1$ paths, and $|C \backslash R|=k-2 t+s$, thus

$$
d\left(u_{1}\right)+d\left(u_{2}\right) \leq 2|X|+d_{H}\left(u_{1}\right)+d_{H}\left(u_{2}\right) \leq 2\left(|H|-1+\frac{|R|-r+t-s+1}{2}+k-2 t+s\right) .
$$

Now concentrate on $v_{1}^{+}$and $v_{2}^{-}$. There cannot be two consecutive vertices in $R \backslash v_{1}^{+} C v_{2}^{-}$, such that one is adjacent to $v_{1}^{+}$and the other adjacent to $v_{2}^{-}$, otherwise the whole segment $v_{1}^{+} C v_{2}^{-}$could be inserted between those two vertices, and a longer cycle through $u_{1}$ could be found. Thus,

$$
d\left(v_{1}^{+}\right)+d\left(v_{2}^{-}\right) \leq 2\left(r-1+\frac{|R|-r+1+t-s}{2}+k-2 t+s+n-|C|-|H|\right) .
$$

But now,

$$
\begin{gathered}
2(n+k-t) \leq d\left(v_{1}^{+}\right)+d\left(u_{1}\right)+d\left(v_{2}^{-}\right)+d\left(u_{2}\right) \\
\leq 2(n+k-t-1+|R|+k-2 t+s-|C|)=2(n+k-t-1),
\end{gathered}
$$

a contradiction. Therefore, there can be at most one vertex adjacent to $H$ in each $R^{i}$.

To prove Theorem 6, observe that the degree condition forces $G$ to be complete or $(k-t+s+1)$-connected. If $G$ is complete we are done. So we may assume that $G$ is $(k-t+s+1)$-connected. Since $|C-R|=k-2 t+s$, there are at least $t+1$ vertices adjacent to $H$ in $R$. Thus, there exists an $R^{i}$ with two such vertices, a contradiction proving Theorem 6.

To prove Theorem 8 , we first prove the following claim.

Claim $2 H$ is the only component of $G-C$.

Otherwise, let $H^{\prime}$ be a different component, let $v_{1} \in H, v_{2} \in H^{\prime}$. For $i=1,2$, let

$$
\begin{aligned}
a_{i} & =\left|\left\{v \in N\left(v_{i}\right) \cap(C \backslash L)\right\}\right|, \\
b_{i} & =\mid\left\{v \in N\left(v_{i}\right): v=x_{j} \text { or } v=y_{j} \text { for some } j \text { with } x_{j} \neq y_{j}\right\} \mid, \\
c_{i} & =\mid\left\{v \in N\left(v_{i}\right): v=x_{j}=y_{j} \text { for some } j\right\} \mid .
\end{aligned}
$$

We know that $a_{i}+b_{i}+2 c_{i} \leq t$, since by Claim $1, v_{i}$ can have at most one neighbor in each $R_{j}$. Further, $b_{i} \leq 2(t-s)$. Thus,

$$
\begin{aligned}
2 d\left(v_{1}\right) \leq 2\left(|H|-1+k-2 t+s+a_{1}+b_{1}+c_{1}\right) & \\
=2|H|+k+a_{1}+k-t-2+\left(b_{1}-2(t-s)\right)+ & \left(a_{1}+b_{1}+2 c_{1}-t\right) \\
& \leq 2|H|+k+a_{1}+k-t-2 .
\end{aligned}
$$

Similarly,

$$
2 d\left(v_{2}\right) \leq 2\left|H^{\prime}\right|+k+a_{2}+k-t-2
$$

Therefore,

$$
n+k-\frac{t+3}{2} \leq d\left(v_{1}\right)+d\left(v_{2}\right) \leq|H|+\left|H^{\prime}\right|+k+\frac{a_{1}+a_{2}}{2}+k-t-2 \leq n+k-t-2,
$$

a contradiction, proving the claim.

The degree condition forces $G$ to be complete or $\left(k-\frac{t-1}{2}\right)$-connected. If $G$ is complete we are done. So we may assume that $G$ is $\left(k-\frac{t-1}{2}\right)$-connected. Since $|C-R|=k-2 t+s$, there are at least $\frac{3 t+1}{2}-s$ neighbors of $H$ in $R$. 
Claim 3 For some $i, 1 \leq i \leq t$, the following is true: $x_{i}=y_{i}$ and $H$ has two neighbors in $y_{i-1} C x_{i+1}^{-} \backslash x_{i}$.

Let $h_{i}$ count the number of neighbors of $H$ in $y_{i-1} C x_{i} \cup y_{i} C x_{i+1}^{-}$. We know that $h_{i} \in\{0,1,2\}$ for all $1 \leq i \leq t$. Further, $\sum_{i} h_{i} \geq 3 t+1-2 s-(t-s)$, since the sum counts every neighbor of $H$ in $\left\{x_{i}: x_{i} \neq y_{i}\right\}$ once and all other neighbors of $H$ in $R$ twice. Thus, at least $(t-s)+1$ of the $h_{i}$ are equal to 2 . Therefore, $h_{i}=2$ for some $i$ with $x_{i}=y_{i}$. The vertex $x_{i}$ cannot be one of the two neighbors of $H$ by Claim 1, establishing the claim.

Let $i$ be as in Claim 3, let $y \in y_{i-1} C x_{i}^{-}$and $z \in y_{i}^{+} C x_{i+1}^{-}$be the two neighbors of $H$. If $y^{+} z^{+} \in E$, then $y H z C^{-} y^{+} z^{+} C y$ is a longer cycle. Thus, $y^{+} z^{+} \notin E$ and, since $y^{+}$and $z^{+}$ are not in $N(H)$,

$$
|C| \geq 2+\frac{d\left(y^{+}\right)+d\left(z^{+}\right)}{2}>\frac{n+k}{2}-\frac{t}{4}+1 .
$$

This implies that

$$
|R|=|C|-k+2 t-s>\frac{n-k}{2}>5 k .
$$

Now let $u \in H, v \in C-N(H)$. Then

$d(v) \geq n+k-\frac{t+3}{2}-d(u) \geq n+k-\frac{t+3}{2}-(k-2 t+s)-t-|H| \geq|C|-1-s+\frac{t-1}{2}$.

Therefore, $v$ is adjacent to all but at most $\frac{s}{2}$ vertices on $C$.

For the final contradiction we differentiate two cases.

Case 1 Suppose $y^{+} \neq x_{i}$ or $z^{+} \neq x_{i+1}$.

Let $w \in\left\{y^{+}, z^{+}\right\}-\left\{x_{i}, x_{i+1}\right\}$. Let $N=N\left(x_{i}\right) \cap N\left(x_{i+1}\right) \cap N(w)$. Since none of the vertices $x_{i}, x_{i+1}, w$ is adjacent to $H$, each is adjacent to all but at most $\frac{s}{2}$ vertices of the cycle. Thus, $|N| \geq|C|-\frac{3 s}{2}$.

Claim 4 For some $j,\left|N \cap y_{j} C x_{j+1}\right| \geq 4$.

Otherwise,

a contradiction.

$$
5 k<|R| \leq 3 t+|R|-|N| \leq 3 t+\frac{3 s}{2},
$$

Let $j$ be as in the last claim, and let $v_{1}, v_{2}, v_{3}, v_{4} \in N \cap y_{j} C x_{j+1}$ be the first four of these vertices in that order.

If $v_{4} \in y^{+} C x_{i}$, define a new cycle as follows: $C^{\prime}=z C^{-} v_{4} x_{i+1} C y H z$.

If $v_{4} \in z^{+} C x_{i+1}$, let $C^{\prime}=z C^{-} x_{i} v_{4} C y H z$.

Otherwise observe that there is at most one neighbor $x$ of $H$ in $v_{1} C v_{4}$.

For $j \neq i$, define the new cycle $C^{\prime}$ as follows:

If $x \in v_{1} C v_{2}$, let $C^{\prime}=z C^{-} x_{i} v_{3} x_{i+1} C v_{2} w v_{4} C y H z$.

If $x \in v_{3} C v_{4}$, let $C^{\prime}=z C^{-} x_{i} v_{2} x_{i+1} C v_{1} w v_{3} C y H z$.

Otherwise, let $C^{\prime}=z C^{-} x_{i} v_{2} C v_{3} x_{i+1} C v_{1} w v_{4} C y H z$.

For $i=j$, a very similar construction works:

let $C^{\prime}=z C^{-} v_{4} w v_{1} C^{-} x_{i} v_{2} C v_{3} x_{i+1} C y H z$.

In any case, no vertex in $C-C^{\prime}$ is adjacent to $H$, so all of them have high degree to $C$ and thus high degree to $R \cap C^{\prime}$. Therefore, we can insert them one by one into $C^{\prime}$ creating a longer cycle, a contradiction, completing Case 1. 
Case 2 Suppose $y^{+}=x_{i}, z^{+}=x_{i+1}$.

Let $N^{\prime}=N\left(x_{i}\right) \cap N\left(x_{i+1}\right)$. Then $\left|N^{\prime}\right| \geq|C|-s$.

Claim 5 For some $l,\left|N^{\prime} \cap y_{l} C x_{l+1}\right| \geq 5$.

Otherwise,

$$
5 k<|R| \leq 4 t+|R|-\left|N^{\prime}\right| \leq 4 t+s
$$

a contradiction.

Let $l$ be as in the last claim, and let $z_{1}, z_{2}, z_{3}, z_{4}, z_{5} \in N^{\prime} \cap y_{l} C x_{l+1}$ be the first five of these vertices in that order. At most one of them is adjacent to $H$, say $z_{2}$. Now a very similar argument as in the last case gives the desired contradiction, just replace $x_{i}$ by $z_{1}, x_{i+1}$ by $z_{5}$, and $w$ by $z_{4}$. One possible cycle would then be (for $l<j<i$ ): $C^{\prime}=z C^{-} x_{i} z_{2} C z_{3} x_{i+1} C z_{1} v_{2} C v_{3} z_{5} C v_{1} z_{4} v_{4} C y H z$.

Theorem 9 If $s=t=k \geq 3$ or $0 \leq s<t<k$, and $G$ is a graph of order $n \geq$ $\max \left\{178 t+k, 8 t^{2}+k\right\}$ with

$$
\sigma_{2}(G) \geq \begin{cases}n+k-3 & \text { if } s=0 \\ n+k+s-4 & \text { if } 0<2 s \leq t \\ n+k+\frac{t-9}{2} & \text { if } 2 s>t\end{cases}
$$

then $G$ is strongly $(k, t, s)$-ordered.

Proof of Theorem 9. To simplify the proof, we will first use an induction argument on $k$ : The statement is obviously true for the base cases $(s=0, t=1, k=2)$ and $(s=t=k=3)$, since $G$ then is 2-connected. Suppose the statement is true for all $k \leq k_{0}$. We need to show the statement for $k=k_{0}+1$. So, let $G$ be a graph of order $n \geq \max \left\{178 t+k, 8 t^{2}+k\right\}$ satisfying the degree condition for some triple $(k, t, s)$. We need to show that for any $(k, t, s)$-linear forest $L$ in $G$, we can find a cycle passing through it in the designated order and direction. Let $L$ be such a forest. Delete all inner vertices of the paths from $V(G)$, and replace the paths by edges to create a new graph $G^{\prime}$ and a new linear forest $L^{\prime}$. If there are any paths of three or more vertices in $G$, this will reduce the order of $G$ and the order of $L$. Finding a cycle in $G^{\prime}$ through $L^{\prime}$ yields a cycle in $G$ through $L$. Since $k^{\prime}=2 t-s, n^{\prime}=n-\left(k-k^{\prime}\right) \geq \max \left\{178 t+k^{\prime}, 8 t^{2}+k^{\prime}\right\}$, and

$$
\sigma_{2}\left(G^{\prime}\right) \geq \sigma_{2}(G)-2\left(k-k^{\prime}\right) \geq \begin{cases}n^{\prime}+k^{\prime}-3 & \text { if } s=0 \\ n^{\prime}+k^{\prime}+s-4 & \text { if } 0<2 s \leq t \\ n^{\prime}+k^{\prime}+\frac{t-9}{2} & \text { if } 2 s>t\end{cases}
$$

there is such a cycle in $G^{\prime}$ if $k^{\prime}<k$, by the induction hypothesis. Thus, we may assume that $k^{\prime}=k$, and so $L=L^{\prime}$, meaning that $L$ consists only of paths with one or two vertices.

Claim 1 G has a t-linked subgraph $H$. 
All vertices of $G$ with $d(v)<\frac{n}{2}$ have to be adjacent. If there are at least $2 t$ of them, this clique is $H$. Otherwise $|E(G)| \geq(n-2 t) \frac{n}{4} \geq 44 t n$, which implies by Corollary 3 that $G$ contains a $22 t$-connected subgraph $H$. By Theorem $1, H$ is $t$-linked.

Claim $2 G$ is t-linked (and thus $(2 t-s, t, s)$-ordered) or $V(G)=V(A) \cup V(B)$, where $|A| \leq|B|+2 t-1, B$ is t-linked, and $A$ is either t-linked or complete.

If $G$ is $2 t$-connected, then $G$ is $t$-linked by Lemma 1 . So assume there is a cut set $K$ with $|K|<2 t$. Let $A^{\prime}$ and $B^{\prime}$ be two components of $G-K$ with $\left|A^{\prime}\right| \leq\left|B^{\prime}\right|$. Let $v \in A^{\prime}, w \in B^{\prime}$. Then

$$
n+2 t-s-3 \leq d(v)+d(w) \leq\left|A^{\prime}\right|+\left|B^{\prime}\right|+2|K|-2 \leq n+2 t-3,
$$

so $u$ and $v$ can miss a total of at most $s$ possible adjacencies. Since $\left|B^{\prime}\right|>\frac{n}{2}-t$, this ensures $B^{\prime}$ to be $22 t$-connected and thus $t$-linked. If $A^{\prime}$ is complete, we are done. Otherwise, the degree sum condition insures $\left|A^{\prime}\right| \geq \frac{n-2 t-s+1}{2}$, so $A^{\prime}$ is $22 t$-connected and thus $t$-linked. To find $A$ and $B$, we now partition the vertices of $K$ as follows one-by-one: Add any vertex $u \in K$ with degree $d_{B^{\prime}}(u) \geq 2 t-1$ to $B^{\prime}$, and add the remaining vertices to $A^{\prime}$. The result will be as desired, as can be seen step by step: If $u$ has high $(\geq 2 t-1)$ degree to $B^{\prime}$, adding it to $B^{\prime}$ will leave $B^{\prime} t$-linked by Lemma 2 . If $u$ has low degree to $B^{\prime}$, it must be either adjacent to all of $A^{\prime}$ or have high degree to $A^{\prime}$ by the degree sum condition. In both cases, $A^{\prime}$ stays complete (if $\left|A^{\prime}\right|<2 t$ ), or $A^{\prime}$ stays $t$-linked (note that a complete graph on $2 t$ vertices is $t$-linked), again by Lemma 2 . This proves the claim.

\section{Case 1 Suppose $t<2 s$.}

First, we may assume that $t \geq 3$. Otherwise, $t=s \leq 2$, and there is nothing to prove. We will use $A^{\prime}$ and $B^{\prime}$ as defined in the proof of Claim 2 above. There is a vertex $v \in B^{\prime}$ with $d_{A}(v)=0$ : For every vertex $w \in A^{\prime}$ we have $d_{B^{\prime}}(w)=0$, and for every $w \in A \cap K$ we have $d_{B^{\prime}}(w) \leq 2 t-2$. Since there are at most $2 t-1$ vertices in $A \cap K$, at most $(2 t-2)(2 t-1)<\left|B^{\prime}\right|$ vertices can have $d_{A}(v)>0$.

Therefore, by the degree sum condition, we have $d_{B}(w) \geq 2 t-s+\frac{t-5}{2}$ for every $w \in A$. Let $L=\left\{x_{1} y_{1}, x_{2} y_{2}, \ldots, x_{t} y_{t}\right\}$, where $x_{i}=y_{i}$ if the path is a singleton, and all paths are directed from $x_{i}$ to $y_{i}$ (remember: all paths are either edges or singletons by the induction hypothesis). We need to find paths from $y_{i}$ to $x_{i+1}$. Let

$$
\begin{aligned}
& L_{A}=L \cap A, \\
& L_{B}=L \cap B, \\
& L_{A}^{\prime}=\left\{x_{i} \in L_{A} \mid y_{i-1} \in L_{B}\right\} \cup\left\{y_{i} \in L_{A} \mid x_{i+1} \in L_{B}\right\}, \\
& L_{B}^{\prime}=\left\{x_{i} \in L_{B} \mid y_{i-1} \in L_{A}\right\} \cup\left\{y_{i} \in L_{B} \mid x_{i+1} \in L_{A}\right\}, \\
& S_{A}=\left\{x_{i} \in L_{A} \mid y_{i-1} \in L_{B}\right\} \cap\left\{y_{i} \in L_{A} \mid x_{i+1} \in L_{B}\right\}, \\
& S_{B}=\left\{x_{i} \in L_{B} \mid y_{i-1} \in L_{A}\right\} \cap\left\{y_{i} \in L_{B} \mid x_{i+1} \in L_{A}\right\} .
\end{aligned}
$$

By these definitions we get

$$
\left|L_{A}^{\prime}\right|+\left|S_{A}\right|=\left|L_{B}^{\prime}\right|+\left|S_{B}\right| .
$$

For $x_{i} \in L_{A}^{\prime}$, let $N^{\prime}\left(x_{i}\right)=\left(N\left(x_{i}\right) \cap B\right)-\left(L-\left\{y_{i-1}\right\}\right)$.

For $y_{i} \in L_{A}^{\prime}$, let $N^{\prime}\left(y_{i}\right)=\left(N\left(y_{i}\right) \cap B\right)-\left(L-\left\{x_{i+1}\right\}\right)$. 
For $X \subset L_{A}^{\prime}$, let

$$
N^{\prime}(X)=\bigcup_{x_{i} \in X} N^{\prime}\left(x_{i}\right) \cup \bigcup_{y_{i} \in X} N^{\prime}\left(y_{i}\right)
$$

For $t=s=3$, there is nothing to prove. For $t=3, s=2$, we get for every nonempty $X \subset L_{A}^{\prime}$,

$$
\left|N^{\prime}(X)\right| \geq 3-\left|L_{B}\right|+|X|+\left|X \cap S_{A}\right| \geq|X|+\left|X \cap S_{A}\right|
$$

For $t \geq 4$ we get for every nonempty $X \subset L_{A}^{\prime}$,

$$
\begin{aligned}
\left|N^{\prime}(X)\right| & \geq 2 t-s+\frac{t-5}{2}-\left|L_{B}\right|+|X|+\left|X \cap S_{A}\right|-\left|S_{B}\right| \\
& =|X|+\left|X \cap S_{A}\right|+\left|L_{A}\right|-\left|S_{B}\right|+\frac{t-5}{2} \\
& \geq|X|+\left|X \cap S_{A}\right|+\left|L_{A}^{\prime}\right|-\left|S_{B}\right|+\frac{t-5}{2} \\
& =|X|+\left|X \cap S_{A}\right|+\frac{\left|L_{A}^{\prime}\right|-\left|S_{B}\right|+\left|L_{B}^{\prime}\right|-\left|S_{A}\right|}{2}+\frac{t-5}{2} \\
& \geq|X|+\left|X \cap S_{A}\right|+\frac{t-5}{2} .
\end{aligned}
$$

Thus, $\left|N^{\prime}(X)\right| \geq|X|+\left|X \cap S_{A}\right|$, and thus by Hall's Theorem, we can find disjoint neighbors for all $x_{i}, y_{i} \in L_{A}^{\prime}$ in $N^{\prime}\left(x_{i}\right)$ or $N^{\prime}\left(y_{i}\right)$, respectively. Using that $B$ is $t$-linked and that $A$ is $t$-linked or complete, we can now find the desired cycle.

Case 2 Suppose $s=0$.

The degree condition forces $G$ to be $(2 t-1)$-connected. If $G$ is $2 t$-connected, then it is $t$-linked and we are done. If $G$ has a cut set $K$ of size $2 t-1$, the degree condition forces $G-K$ to consist of two complete components $A^{\prime}$ and $B^{\prime}$, both of which are adjacent to all ertices in $K$. It is easy to see that such a graph is $t$-linked.

Case 3 Suppose $0<s \leq t / 2$.

The degree condition forces $G$ to be $(2 t-2)$-connected. If $G$ is $2 t$-connected, then it is $t$-linked and we are done. If $G$ has a cut set $K$ of size $2 t-2$, the degree condition forces $G-K$ to consist of two complete components $A^{\prime}$ and $B^{\prime}$, both of which are adjacent to all vertices in $K$. It is easy to see that such a graph is $(2 t-s, t, s)$-ordered. If $K$ has size $2 t-1, G$ has a very similar structure. Again, it is straightforward to verify the claim.

Theorem 10 If $0 \leq s \leq t \leq k$, and $G$ is a graph of order $n \geq \max \left\{178 t+k, 8 t^{2}+k\right\}$ with

$$
\sigma_{2}(G) \geq \begin{cases}n+k-3 & \text { if } s=0, t \geq 3 \\ n+k+s-4 & \text { if } 0<2 s \leq t, t \geq 3 \\ n+k+\frac{t-9}{2} & \text { if } 2 s>t \geq 3 \\ n+k-2 & \text { if } s \leq 1, t=2 \\ n+k-1 & \text { if } s=0, t=1 \\ n & \text { if } s=t \leq 2\end{cases}
$$

then $G$ is strongly $(k, t, s)$-ordered hamiltonian. 
Proof: Apply Theorem 6 and Theorem 9.

\section{Sharpness}

Theorem 6 is sharp for $s=0$, illustrated by the following graph: Let $A=K_{\frac{n+k-t-1}{2}}$, and $B$ be a set of $\frac{n-k+t+1}{2}$ isolated vertices. Add all edges between $A$ and $B$. For $n$ sufficiently large, $G$ is strongly $(k, t, s)$-ordered, and $\sigma_{2}(G)=n+k-t-1$. But $G$ is not strongly $(k, t, s)$-ordered hamiltonian, since no hamiltonian cycle can contain a $(k, t, s)$-linear forest $L$ which completely lies inside $A$ : Every hamiltonian cycle has exactly $k-t-1$ edges in $A$, one edge less than $L$.

The following graph shows sharpness of Theorem $9, s=0$. Let $G$ consist of three complete graphs: $A=K_{\frac{n-k+2}{2}}, K=K_{k-2}, B=K_{\frac{n-k+2}{2}}$. Add all edges between $A$ and $K$ and all edges between $K$ and $B$. The degree sum condition is just missed, but $G$ is not $(k, t, 0)$-ordered: Let $x_{1} \in A, y_{t} \in B,\left\langle L-\left\{x_{1}, y_{t}\right\}\right\rangle=K$.

The following graph shows sharpness of Theorem $9, t \geq 2 s \geq 2$. Let $G$ consist of four complete graphs: $S=K_{s}, T=K_{k-s}, A=K_{2 s-1}, B=K_{n-k-2 s+1}$. Add all edges from $A$, all edges between $T$ and $B$. For every vertex $s_{i} \in S$, pick two vertices $u_{i}, v_{i} \in T$. Add all edges between $S$ and $T$ but the edges $s_{i} u_{i}, s_{i} v_{i}$. We have $\sigma_{2}(G)=n+k+s-5$, but if we pick $V(L)=V(S) \cup V(T)$, such that $x_{2 i}=y_{2 i}=s_{i}, x_{2 i+1}=u_{i}, y_{2 i-1}=v_{i}$ for all $i \leq s$, there is no cycle passing through $L$ in the designated order and direction.

The following graph shows sharpness of Theorem $9,2 s>t$. Let $G$ consist of four complete graphs: $S=K_{\left\lceil\frac{t}{2}\right\rceil}, T=K_{k-\left\lceil\frac{t}{2}\right\rceil}, A=K_{t-1}, B=K_{n-k-2 s+1}$. Add all edges from $A$, all edges between $T$ and $B$. For every vertex $s_{i} \in S$, pick two vertices $u_{i}, v_{i} \in T$, with the exception that $v_{i+1}=u_{i}$ for $1 \leq i \leq s-\left\lceil\frac{t}{2}\right\rceil$. Add all edges between $S$ and $T$ but the edges $s_{i} u_{i}, s_{i} v_{i}$. We have $\sigma_{2}(G)=n+k+\left\lfloor\frac{t}{2}\right\rfloor-5$, but if we pick $V(L)=V(S) \cup V(T)$, such that $x_{2 i}=y_{2 i}=s_{i}, x_{2 i+1}=u_{i}, y_{2 i-1}=v_{i}$ for all $i \leq\left\lceil\frac{t}{2}\right\rceil$, there is no cycle passing through $L$ in the designated order and direction.

\section{Note added in proofs}

Very recently, Thomas and Wollan [8] have improved the bound in Theorem 1 to the following.

Theorem 11 If a graph $G$ is $2 k$-connected and has at least $5 k|V(G)|$ edges, then $G$ is $k$-linked.

Corollary 12 Every 10k-connected graph is k-linked.

Using these results in place of Theorem 1 will improve some of the bounds on $n$. 


\section{References}

[1] Bollobás, B. and Thomason, A., Highly Linked Graphs, Combinatorics, Probability, and Computing, 1993, 1-7.

[2] Faudree, J.R., Faudree, R.J., Gould, R.J., Jacobson, M.S., and Lesniak, L., On kOrdered Graphs, J. Graph Theory, 35 (2000), 69-82.

[3] Faudree, R.J., Gould, R.J., Kostochka, A., Lesniak, L., Schiermeyer, I., and Saito, A., Degree Conditions for $k$-ordered hamiltonian graphs, J. Graph Theory, 42 (2003), 199210.

[4] Hu, Z., Tian, F. and Wei, B. Long cycles through a linear forest, JCT B 82(2001), no. $1,67-80$.

[5] Kierstead, H., Sarkozy, G., and Selkow, S., On k-Ordered Hamiltonian Graphs, J. Graph Theory, 32 (1999), 17-25.

[6] Mader, W., Existenz von n-fach zusammenhängenden Teilgraphen in Graphen genügend grosser Kantendichte, Abh. Math. Sem. Univ. Hamburg, 37 (1972), 86-97.

[7] Ng, L. and Schultz, M., k-Ordered Hamiltonian Graphs, J. Graph Theory, 24 (1997), $45-57$.

[8] Thomas, R. and Wollan, P., An Improved Edge Bound for Graph Linkages, preprint. 